OPEN ACCESS

Edited by: Farid Amirouche,

University of Illinois at Chicago,

United States

Reviewed by:

Karthikeyan Chinnakkannu,

Plancher Orthopaedics and Sports Medicine, United States George Murrell,

University of New South Wales, Australia

*Correspondence: Antonio Gigante scienza.clinortop@gmail.com

Specialty section: This article was submitted to Orthopedic Surgery,

a section of the journal

Frontiers in Surgery

Received: 30 October 2020 Accepted: 01 November 2021

Published: 06 December 2021

Citation:

Luciani P, Farinelli L, De Berardinis L and Gigante $A$ (2021) The

Arthroscopic Intra-Articular

Stabilization of the Shoulder for Irreparable Rotator Cuff Tear: A New Technique Proposal.

Front. Surg. 8:624100.

doi: 10.3389/fsurg.2021.624100

\section{The Arthroscopic Intra-Articular Stabilization of the Shoulder for Irreparable Rotator Cuff Tear: A New Technique Proposal}

\author{
Pierfrancesco Luciani, Luca Farinelli, Luca De Berardinis and Antonio Gigante* \\ Clinical Orthopaedics, Department of Clinical and Molecular Sciences, UniversitàPolitecnicadelle Marche, Ancona, Italy
}

Background: Superior capsular reconstruction (SCR) has shown good results in the management of irreparable rotator cuff tears due to the depressive effect on the humeral head, but it is a technically demanding and expensive procedure.

Purpose: We hypothesized that an intra-articular neoligament that prevents the superior translation of the humeral head could give similar results in terms of the superior translation of humerus (STH) and range of motion (ROM).

Study Design: To compare our proposed technique and the SCR, we conducted a biomechanical study on 10 porcine shoulders in a custom shoulder testing system.

Methods: STH and total rotational ROM were quantified in the following four scenarios: (1) when the rotator cuff was intact, (2) after cutting the supraspinatus tendon, (3) after the reconstruction of the superior capsule by long head of the biceps tendon (LHB), and (4) after an arthroscopic intra-articular stabilization by an intra-articular graft. Our proposed technique provides the creation of a humeral and glenoid tunnel, the passage of a graft through these tunnels under arthroscopic guidance, and the graft fixation in the two tunnels. We analyzed the STH and total ROM in each scenario.

Results: With respect to the STH, we reported that the present proposed technique is characterized by a significant reduction of superior translation at 0 and $45^{\circ}$ compared to scenario 2. In addition, the comparison between our proposed technique and SCR showed a significant difference of the $\mathrm{STH}$ at $\mathrm{O}^{\circ}$ of abduction. Total rotational ROMs of the two tenchinques were similar to scenario 2. Therefore, the use of an intra-articular ligament that prevents the STH can restore shoulder stability in irreparable rotator cuff injuries at both 0 and $45^{\circ}$ of glenohumeral abduction without apparently limiting the total rotational $\mathrm{ROM}$.

Conclusion: Our proposed technique could be an important treatment option in irreparable rotator cuff tears, especially in patients under 65 years in whom reverse shoulder arthroplasty (RSA) has shown poor results and many complications.

Keywords: rotator cuff tear, technique, arthroscopic stabilization, neoligament, shoulder 


\section{INTRODUCTION}

Massive rotator cuff tears could determine significant pain and disability of the shoulder (1). These lesions are often characterized by chronic inelastic retraction or significant muscle fat degeneration that can preclude successful repair (2-7). Treatment options for massive and irreparable rotator cuff injuries depend on a multitude of factors including age of the patient, activity level and expectation, degree of joint arthropathy, and the extent of disability caused by the injury (8). In these lesions, surgical options include arthroscopic debridement, biceps tenotomy/tenodesis, partial cuff repair, interposition grafts, subacromial balloon spacers, tendon transfers, superior capsular reconstruction (SCR), and reverse shoulder arthroplasty (RSA) (9, 10). Although RSA

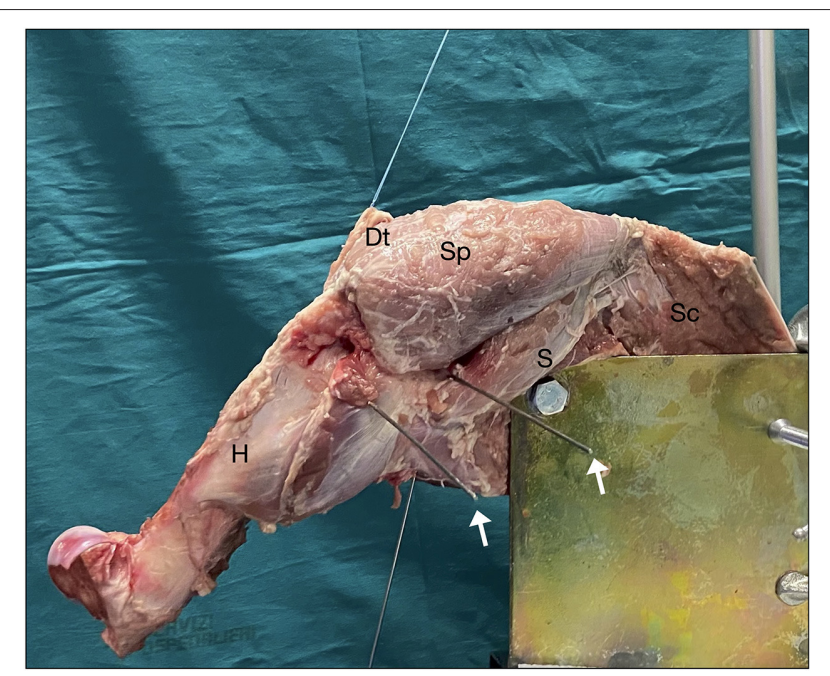

FIGURE 1 | Shoulder fixed to the system prepared for test. The deltoid tendon (Dt) is basted for the application of forces. White arrows: K-wires located as explained in the text. Sp, sopraspinatus; S, subscapularis; SC, scapula; H, humerus. has shown excellent results in irreparable rotator cuff tears in patients over 65 years, in younger patients, it has not been as successful with complication rates up to $50 \%$ (11-13) and with unsatisfactory clinical outcomes (14). The superior translation of the humeral head following massive rotator cuff tears causes impaired glenohumeral joint mechanics, shoulder dysfunction, and, in the long term, the development of glenohumeral arthrosis. Studies on SCR have shown that returning the humeral head to its original position restores joint biomechanics and improves clinical outcomes $(15,16)$.

Nowadays, SCR is to be considered a technically demanding and onerous procedure due to the cost of the allograft and the devices used to fix it and the prolonged operating time (17).

Therefore, recent biomechanical studies have used the long head of the biceps tendon (LHB) for the superior capsular reconstruction showing the stability of the glenohumeral joint (18-21).

In this study, given the excellent clinical outcomes obtained by the SCR due to the depressant effect on the humeral head, we hypothesized that similar results can be obtained using an intra-articular graft that prevents the superior translation of the humeral head and at the same time, it is able to balance the pairs of forces of the anterior and posterior cuff. Therefore, we proposed an arthroscopic intra-articular stabilization of the shoulder named as the AISS technique. The aim of this biomechanical study was to compare the STH and total range of motion (ROM) of porcine shoulder in case of irreparable rotator cuff tears treated by our proposed AISS technique and the SCR technique with LHB tendon described by Chillemi et al. (17) known as the Arthroscopic Biceps Chillemi (ABC) technique.

\section{MATERIALS AND METHODS}

\section{Specimen Preparation}

This study was conducted on 10 frozen adult porcine shoulders. Specimens were thawed at room temperature on the night before dissection and testing. The skin, subcutis, and muscles were removed from each sample, while the supraspinatus, teres
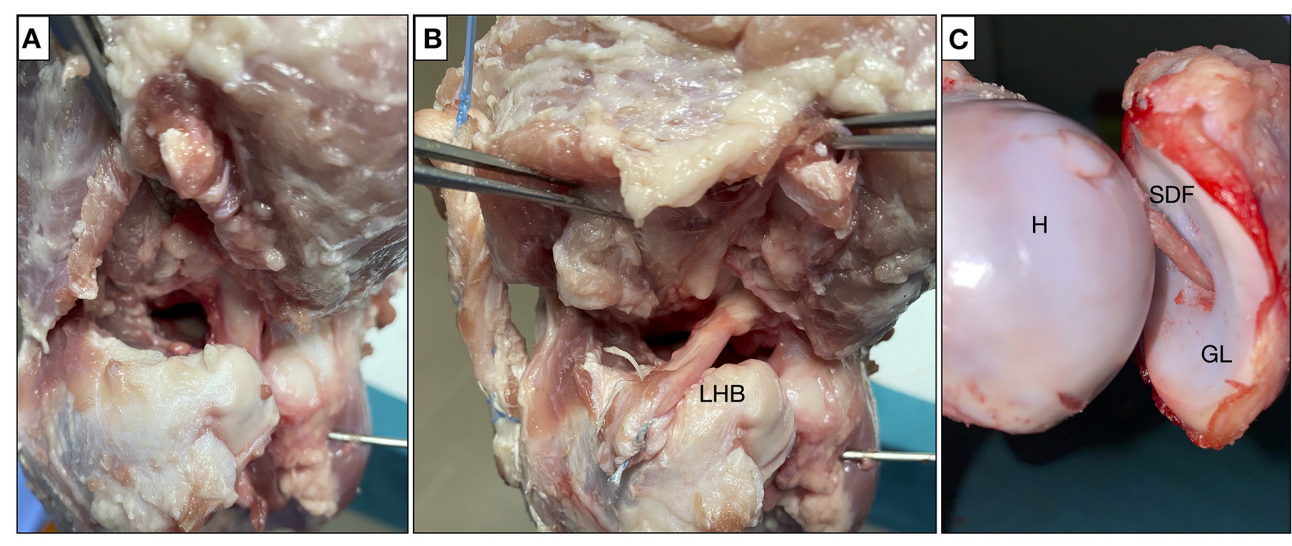

FIGURE 2 | Testing scenario: (A) complete tear of the supraspinatus; (B) SCR with ABC technique; and (C) stabilization with the AISS technique. SCR, superior capsular reconstruction; ABC, Arthroscopic Biceps Chillemi; AISS, arthroscopic intra-articular stabilization of the shoulder; LHB, long head biceps tendon; SDF, superficial digital flexor tendon; $\mathrm{H}$, humerus; GL, glena. 
minor, infraspinatus, subscapularis, teres major, coracobrachialis muscles, and the deltoid distal tendon insertion were preserved. A No. 2 FiberWire suture (Arthrex Incorporation, Naples, Florida) was used to tie a krackow locking-running stitch to the tendinous insertion for muscle loading during testing. The scapula was fixed to a custom metal plate by using three large bolts (Figure 1). The scapular plate was fixed to a custom testing jig in $20^{\circ}$ of anterior tilt in the sagittal plane. A goniometer was placed at the distal end of the humerus to measure humeral rotation. A torque dynamometric wrench was used to apply a predetermined torque for measuring rotational ROM. Muscle forces were simulated via a fishing line attached to No. 2 FiberWire suture, which was attached directly to the tendon. Adjustable pulleys and a positioning plate were used to approximate physiological muscle force vectors without friction. The authors PL and AG performed all the tests.

\section{Testing Conditions}

Every biomechanical test is performed in each of the following four scenarios:

Scenarion 1: The rotator cuff is intact as control.

Scenarion 2: An irreparable supraspinatus tear model has been made cutting the supraspinatus and superior capsule at the insertion on the greater tuberosity to the glenoid along the anterior and posterior edges of the supraspinatus tendon. To simulate an irreparable rotator cuff tear, the lateral edges of the supraspinatus and superior capsule were retracted to the glenoid (Figure 2A).

Scenarion 3: A SCR was performed by using a LHB tendon as described in the ABC technique (17) (Figure 2B). The graft length was determined to be $15 \mathrm{~mm}$ longer than the length from the superior glenoid to the lateral edge of the greater tuberosity at $30^{\circ}$ of glenohumeral abduction. The proximal insertion of the tendon over the glena was left intact, while on the greater tuberosity the tendon was attached with two anchors (ReelX STT $4.5 \mathrm{~mm}$, Stryker, Kalamazoo, MI). The graft was attached at $30^{\circ}$ of glenohumeral abduction in the scapular plane (equivalent to $45^{\circ}$ of shoulder abduction), as described in the original technique.

Scenarion 4: LHB tendon was removed and intra-articular shoulder arthroscopic stabilization is performed (AISS technique) (Figure 2C).

\section{Surgical Technique}

A proposed surgical technique steps are given in Figure 3. The steps are explained as following.

\section{Humeral Tunnel}

A K-wire was inserted under fluoroscopic control $1 \mathrm{~cm}$ distal to the superior margin of the greater tuberosity with humerus positioned at $0^{\circ}$ of abduction and neutral rotation. The wire must be parallel to the ground, follow the humeral retroversion of $20^{\circ}$, and protrudes on the anatomical surface of the humerus at the height of the superior margin of the glena. At this point, a 5-mm

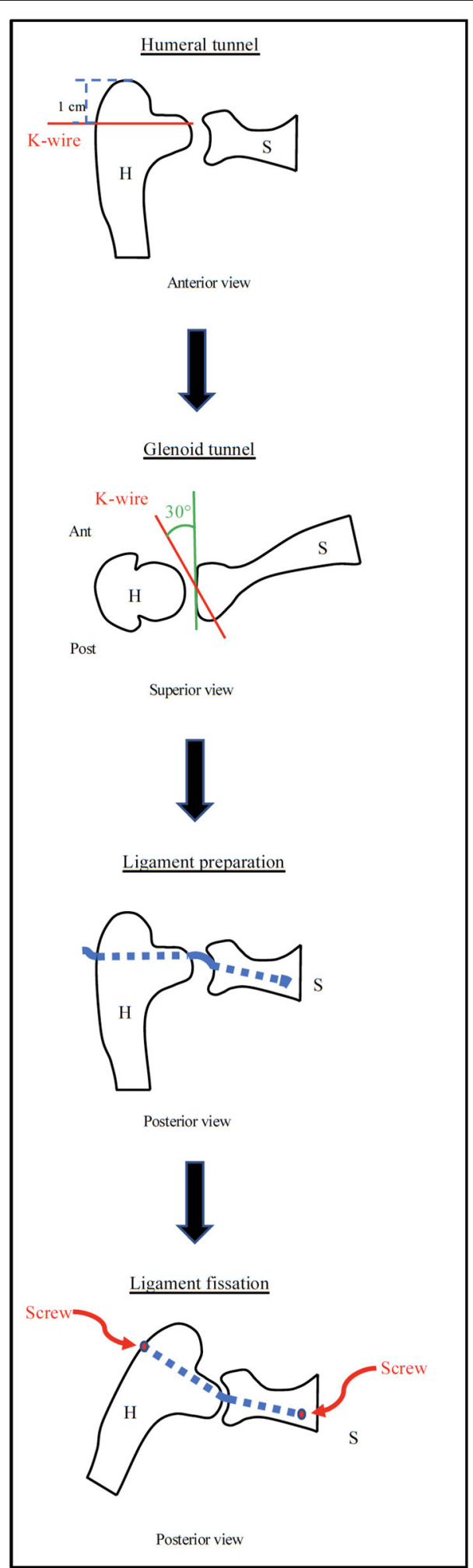

$\mathrm{H}$, humerus; S, scapula;

FIGURE 3 | Diagramatic representation of the AISS technique. 


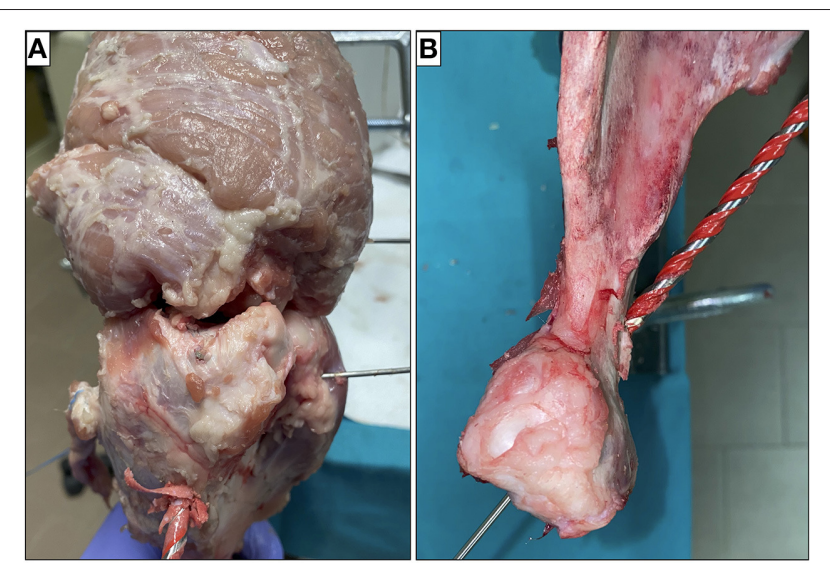

FIGURE 4 | Humerus (A) and glena (B) were drilled on the K-wire guide.

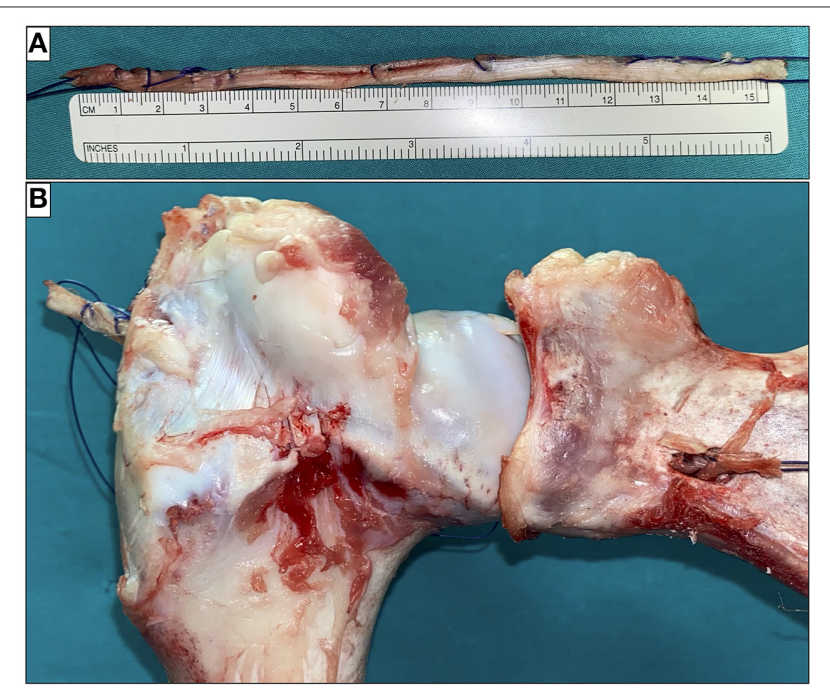

FIGURE 5 | The ligament was basted with absorbable wires (A) and passed through the two tunnels (B).

hole was drilled on the wire guide. The length of the tunnel is approximately $8.5 \mathrm{~cm}$ in porcine shoulder (Figure $4 \mathrm{~A}$ ).

\section{Glenoid Tunnel}

After creating a standard posterior arthroscopic portal (for optics), an anterosuperior arthroscopic portal was performed. Through this portal, a K-wire was pointed at the glenoid fovea. The K-wire was inserted into the glena maintaining an inclination of $30^{\circ}$ with respect to the sagittal plane and $10^{\circ}$ to the axial plane in a posteroinferior direction. The wire will come out at the level of the scapula neck between the lower edge of the neck and the spine of the scapula.

At this point, we proceeded to execute a posterior miniaccess centered on the K-wire. The suprascapular nerve was identified and carefully isolated and a $5-\mathrm{mm}$ hole was drilled on the wire guide. The length of the tunnel is about $3 \mathrm{~cm}$ (Figure 4B).

\section{Ligament Preparation}

A ligament of about $15 \mathrm{~cm}$ by $5 \mathrm{~mm}$ in diameter (obtained from the porcine superficial digital flexor tendon) was basted with absorbable wires (Figure 5A) and was passed before in the humeral tunnel and then in the glenoid tunnel under arthroscopic vision (Figure 5B).

\section{Ligament Fixation}

Many devices could be used for ligament fixation to the glena as a staple or a screw. We have excluded indirect fixations such as Endobutton or TightRope given the shortness of bone tunnel and direct fixations to the glenoid fovea in order not to preclude the future possibility of a reverse prosthesis. In this study, the ligament was fixed to the scapula using a 4-mm screw inserted from a posterior miniaccess taking care not to damage the suprascapular nerve (Figure 6A).

Finally, the ligament was tensioned and fixed to the humerus with a $4-\mathrm{mm}$ interference screw at $30^{\circ}$ of glenohumeral abduction (about $45^{\circ}$ of shoulder abduction) (Figure 6B) as suggested by SCR technique (22).

\section{Measurements}

All the measurements were performed at 0 and $45^{\circ}$ of glenohumeral abduction and with neutral humeral rotation. For each position, it has been used for two different loading conditions: in condition n.1 (named rest condition), the deltoid was subjected to a force of $40 \mathrm{~N}$, while in condition n.2 (named stress condition), the deltoid was subjected to a force of $80 \mathrm{~N}$ because we would simulate the superior translation force in order to evaluate the stability of the shoulder.

The amount and direction of translation relative to the initial position were quantified using $\mathrm{K}$-wires placed on the midpoint of the anterior glenoid region and in the proximal portion of the bicipital groove by a three-dimensional digitizer (Micro-Scribe 3DLX; Immersion Corporation, San Jose, USA); the accuracy of this device was $0.30 \mathrm{~mm}$ according to the manufacturer. The location of the humeral head relative to the glena was recorded under loading conditions N.1 and N.2. To evaluate superior shoulder stability, superior translation of the humerus was calculated by comparing the distance between two wires in the superior-inferior direction under loading condition 1 with that under loading condition 2 in all the positions and cases previously described.

Total rotational ROM was calculated by adding external and internal rotational ROMs using a $360^{\circ}$ digital goniometer (GemRed). Measurements were performed only in the loading condition 1 because when the head of the humerus has migrated superiorly, making extreme movements that could cause ruptures of the ligament, with consequent inconsistent data.

\section{Statistical Analysis}

All the measurements were repeated three times. The data were collected and organized with Excel (Microsoft, Redmond, Washington, USA). Continuous variables were expressed as mean $\pm \mathrm{SD}$. The superior translation of the humerus and the rotational ROM in the four conditions were compared with the Mann-Whitney $U$ test. Statistical analysis were performed using 

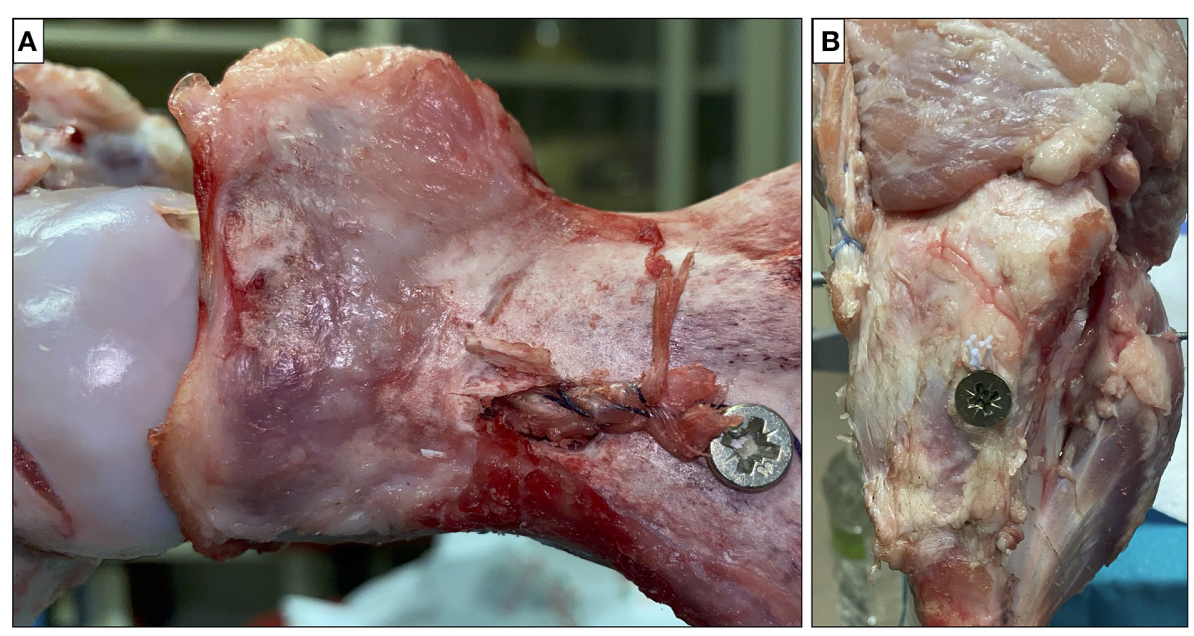

FIGURE 6 | The ligament was fixed at the scapula (A) and humerus (B) with screws.

TABLE 1 | Superior translation of the humerus (STH).

\begin{tabular}{|c|c|c|c|c|c|c|c|c|}
\hline & \multicolumn{2}{|c|}{ Intact } & \multicolumn{2}{|c|}{ Supraspinatus tear } & \multicolumn{2}{|c|}{ ABC technique } & \multicolumn{2}{|c|}{ AISS technique } \\
\hline & $\begin{array}{l}\text { STH, } \\
\text { mm }\end{array}$ & $\begin{array}{c}\text { STH, } \\
\%\end{array}$ & $\begin{array}{l}\text { STH, } \\
\text { mm }\end{array}$ & $\begin{array}{c}\text { STH, } \\
\%\end{array}$ & $\begin{array}{l}\text { STH, } \\
\text { mm }\end{array}$ & $\begin{array}{c}\text { STH, } \\
\%\end{array}$ & $\begin{array}{l}\text { STH, } \\
\text { mm }\end{array}$ & $\begin{array}{c}\text { STH, } \\
\%\end{array}$ \\
\hline $0^{\circ}$ abduction & $1.8 \pm 0.7$ & 100 & $4.3 \pm 0.7^{a}$ & 238 & $2.5 \pm 0.4^{b}$ & 139 & $1.7 \pm 0.4^{b, c}$ & 94 \\
\hline $45^{\circ}$ abduction & $2.10 \pm 6$ & 100 & $4.70 \pm 7^{a}$ & 223 & $2.60 \pm 3^{b}$ & 123 & $20 \pm 5^{b}$ & 95 \\
\hline
\end{tabular}

Values are expressed as mean $\pm S D$. "STH, \%" was calculated by dividing each value by that for the intact condition at the same position.

a Statistically significant difference compared with intact condition $(p<0.05)$.

${ }^{b}$ Statistically significant difference compared with simulated supraspinatus tear $(p<0.05)$.

'Statistically significant difference compared with the Arthroscopic Biceps Chillemi $(A B C)$ repair $(p<0.05)$.

the SPSS (version 21.0; IBM Corporation, Armonk, New York, USA). $p<0.05$ was considered as statistically significant.

\section{RESULTS}

\section{Superior Translation of the Humerus}

The creation of a complete and irreparable lesion of the supraspinatus increases the superior translation of the humerus significantly by $2.5 \mathrm{~mm}$ at $0^{\circ}$ of abduction $(p<0.001)$ and by $2.6 \mathrm{~mm}$ at $45^{\circ}$ of abduction $(p<0.001)$. Both the ABC and the AISS technique make the shoulder more stable compared to scenario 2. No statistically significant differences have been reported compared to the intact cuff. Additionally, it has been observed a reduction of the glenohumeral superior translation of $0.6 \mathrm{~mm}$ with the AISS technique compared to the $\mathrm{ABC}$ one at $45^{\circ}$ of abduction $(p>0.07)$. This difference reaches $0.8 \mathrm{~mm}$ at $0^{\circ}$ of abduction $(p<0.04)$ (Table 1).

\section{Total Rotational ROM}

In scenario N.2, the total rotational ROM of the shoulder increases by 16 and $19^{\circ}$, respectively, at $0^{\circ}(p<0.01)$ and $45^{\circ}$ $(p<0.006)$ of abduction compared to healthy rotator cuff. In scenario N.3, there is a nonsignificant decrease in rotational ROM compared to scenario 2 at 0 and $45^{\circ}$ of abduction. Similarly,
TABLE 2 | Total rotational range of motion (in degrees).

\begin{tabular}{lcccc}
\hline & Intact & SupraspinatusTear & ABC technique & AISStechnique \\
\hline $0^{\circ}$ abduction & $10 \pm 6$ & $26 \pm 5^{\mathrm{a}}$ & $22 \pm 4^{\mathrm{a}}$ & $24 \pm 4^{\mathrm{a}}$ \\
$45^{\circ}$ abduction & $24 \pm 7$ & $43 \pm 5^{\mathrm{a}}$ & $39 \pm 4^{\mathrm{a}}$ & $40 \pm 5^{\mathrm{a}}$ \\
\hline
\end{tabular}

Values are expressed as mean $\pm S D$.

a Statistically significant difference compared with intact condition $(p<0.05)$.

in scenario 4 , it has been observed a decrease of rotational ROM of shoulder of 2 and $3^{\circ}$, respectively, at $0(p>0.46)$ and $45^{\circ}(p>$ $0.35)$ of abduction.

Summarizing, both in the scenario N.3 and N.4, the rotational ROM is significantly greater than that of intact cuff, increasing by 12 and $15^{\circ}$ at $0(p<0.03)$ and $45^{\circ}(p<0.01)$ of abduction with the $\mathrm{ABC}$ technique and 14 and $16^{\circ}$ at $0(p<0.02)$ and $45^{\circ}$ $(p<0.01)$ of abduction with the AISS technique (Table 2$)$.

\section{DISCUSSION}

The most important finding of this study was that the use of an intra-articular graft that prevents superior translation of the humerus could restore shoulder stability in irreparable rotator 
cuff injuries at both the 0 and $45^{\circ}$ of glenohumeral abduction without apparently limiting the total rotational ROM in porcine model. In this biomechanical study, the AISS technique and the intact cuff gave similar results on superior humeral translation. The use of the LHB tendon for the SCR has considerably reduced humeral elevation, but it has not restored the condition given by the intact cuff. This residual superior instability could lead to abrasion and rupture of the LHB tendon after surgery with a higher revision rate than the AISS technique. To date, fascia lata and human dermal graft could be used in SCR (23-26). However, concerns about cost, donor site morbidity in case of autograft, increased surgical time and the significantly elongation of dermal graft during test need to be considered (27). Furthermore, an unstable shoulder works with an altered biomechanics and will undergo arthritic degeneration $(15,16,19)$.

Comparing the two repair techniques, the AISS gave better results on superior humeral translation than the $\mathrm{ABC}$ technique, but this finding was significant only at $0^{\circ}$ of abduction. Previous biomechanical studies on the role of the rotator cuff tendon in shoulder elevation have shown that the rotator cuff tendon is more important in initiation of elevation than at a higher angle of abduction (28). Therefore, restoration of glenohumeral joint stability after the AISS at a lower angle of abduction may occur because the neoligament supports the function of a partially repaired rotator cuff tendon as a superior stabilizer at initiation of shoulder elevation.

Excessive traction of the graft could cause stiff shoulders after surgery. When a complete lesion of the supraspinatus tendon is created, there is a significant increase in the total rotational ROM of the humerus compared to the intact cuff (29). In this study, this parameter in both the techniques remains similar to that of the injured cuff. This suggests that both the interventions should not cause stiffness. Clearly, the porcine shoulder has a lower rotational ROM than humans $\left(141^{\circ}\right.$ in humans vs. $10^{\circ}$ in porcine at $0^{\circ}$ abduction) and, therefore, this finding must be confirmed with human studies by plain radiograph and analysis of ROM (19).

The strength of this biomechanical study is the direct measurement of the superior humeral translation and of the rotational ROM, which is not possible in the living. Moreover, this study is consistent because the exact same force can be applied in the same direction for all the specimens and all the four scenarios were tested in each shoulder.

This study had some limitations. Firstly, it was conducted on porcine shoulders, which have anatomical and biomechanical

\section{REFERENCES}

1. Tokish JM, Thomas CA, Kissenberth MJ, Hawkins RJ. Pseudoparalysis: a systematic review of term definitions, treatment approaches, and outcomes of management techniques. J Shoulder Elbow Surg. (2017) 26:e17787. doi: 10.1016/j.jse.2017.02.024

2. Bedi A, Joshua D, Russell FW, David MD. Massive tears of the rotator cuff. $J$ Bone Joint Surg Am. (2010) 92:1894-908. doi: 10.2106/JBJS.I.01531

3. Goutallier D, Postel J, Bernageau J, Lavau L, Voisin MC. Fatty Muscle Degeneration in Cuff Ruptures. Pre- and postoperative differences from human ones, but porcine models have been commonly used in biomechanical studies for the initial evaluation of rotator cuff reconstruction techniques (30). Muscle loading was static rather than dynamic and this experiment has been performed only in two muscle loading conditions. We did not compare the AISS technique with SCR using fascia lata and human dermal graft because concerns about cost and the significantly elongation over time limit their use.

In addition, the effect of biological healing cannot be evaluated. Lastly, the execution of the humeral tunnel may result in articular cartilage damage in humeral side that could contribute to cartilage degeneration and glenohumeral arthritis. However, it should be pointed out that in massive rotator cuff tears, the cartilage damage is already present. Moreover, the tunnel is small sized and located in the superior part of humeral head.

\section{CONCLUSION}

Our proposed technique could be an important treatment option in irreparable rotator cuff tears, especially in patients under 65 years in whom RSA has shown poor results and many complications. Obviously, further biomechanical studies on human cadaver are mandatory in order to assess the feasibility, safety, and effectiveness of the AISS technique on massive rotator cuff tears.

\section{DATA AVAILABILITY STATEMENT}

The original contributions presented in the study are included in the article/supplementary material, further inquiries can be directed to the corresponding author/s.

\section{ETHICS STATEMENT}

Ethical review and approval was not required for the animal study because the study was conducted on porcine shoulder provided by Butcher's shop.

\section{AUTHOR CONTRIBUTIONS}

AG had the idea, intuition, and supervised. LF, PL, and LD elaborated the project and made the experiment. PL and LD wrote the manuscript. LF corrected the paper. All authors contributed to the article and approved the submitted version. evaluation by CT Scan. Clin Orthop Relat Res. (1994) 304:7883. doi: 10.1097/00003086-199407000-00014

4. Melis B, Nemoz C, Walch G. Muscle fatty infiltration in rotator cuff tears: descriptive analysis of 1688 cases. Orthop Traumatol Surg Res. (2009) 95:31924. doi: 10.1016/j.otsr.2009.05.001

5. Melis B, Wall B, Walch G. Natural history of infraspinatus fatty infiltration in rotator cuff tears. J Shoulder Elbow Surg. (2010) 19:757763. doi: $10.1016 /$ j.jse.2009.12.002

6. Oh JH, Hoon K, Choi J, Kim Y, Hee Oh C. Reliability of the grading system for fatty degeneration of rotator cuff muscles. Clin 
Orthop Relat Res. (2010) 468:1558-64. doi: 10.1007/s11999-0090818-6

7. Oh JH, Hoon K, Yeal Kang J, Hee Oh C, Sik Gong H. Effect of age on functional and structural outcome after rotator cuff repair. Am J Sports Med. (2010) 38:672-8. doi: 10.1177/0363546509352460

8. Boileau P, François B, Laure V, Philip A, Christopher C, Christophe T. Isolated arthroscopic biceps tenotomy or tenodesis improves symptoms in patients with massive irreparable rotator cuff tears. J Bone Joint Surg Am. (2007) 89:747-57. doi: 10.2106/00004623-200704000-00008

9. Kim SJ, Kim SH, Lee SK, Seo JW, Chun Y. Arthroscopic repair of massive contracted rotator cuff tears: aggressive release with anterior and posterior interval slides do not improve cuff healing, and integrity. J Bone Joint Surg Am. (2013) 95:1482-88. doi: 10.2106/JBJS.L.01193

10. Mihata T, McGarry M, Kahn T, Goldberg I, Neo M, Thay Q. Biomechanical effects of acromioplasty on superior capsule reconstruction for irreparable supraspinatus tendon tears. Am J Sports Med. (2016) 44:191-7. doi: 10.1177/0363546515608652

11. Ek E, Neukom L, Catanzaro S, Gerber C. Reverse total shoulder arthroplasty for massive irreparable rotator cuff tears in patients younger than 65 years old: results after five to fifteen years. J Shoulder Elbow Surg. (2013) 22:1199208. doi: 10.1016/j.jse.2012.11.016

12. Guery J, Favard L, Sirveaux F, Oudet D, Mole D, Walch G. Reverse total shoulder arthroplasty. Survivorship analysis of eighty replacements followed for five to ten years. J Bone Joint Surg Am. (2006) 88:17427. doi: $10.2106 / 00004623-200608000-00008$

13. Wall B, Josserand L, O'Connor D, Bradley E, Walch G. Reverse total shoulder arthroplasty: a review of results according to etiology. J Bone Joint Surg Am. (2007) 89:1476-85. doi: 10.2106/00004623-200707000-00011

14. Garcia G, Taylor S, DePalma B, Mahony G, Grawe B, Nguyen J, et al. Patient activity levels after reverse total shoulder arthroplasty: what are patients doing? Am J Sports Med. (2015) 43:2816-21. doi: 10.1177/0363546515597673

15. Mihata $\mathrm{T}$, Lee $\mathrm{T}$, Watanabe $\mathrm{C}$, Fukunishi $\mathrm{K}$, Ohue $\mathrm{M}$, Tsujimura $\mathrm{T}$, et al. Clinical results of arthroscopic superior capsule reconstruction for irreparable rotator cuff tears. Arthroscopy. (2013) 29:459-70. doi: 10.1016/j.arthro.2012.10.022

16. Mihata T, McGarry M, Pirolo J, Kinoshita M, Thay Q. Superior capsule reconstruction to restore superior stability in irreparable rotator cuff tears: a biomechanical cadaveric study. Am J Sports Med. (2012) 40:224855. doi: 10.1177/0363546512456195

17. Chillemi C, Mantovani M, Gigante A. Superior capsular reconstruction of the shoulder: The ABC (Arthroscopic Biceps Chillemi) technique. Eur J Orthop Surg Traumatol. (2018) 28:1215-23. doi: 10.1007/s00590-018-2183-1

18. El-Shaar R, Sandeep S, Nicandri G, Maloney M, Ioloshin I. Superior capsular reconstruction with a long head of the biceps tendon autograft: a cadaveric study. Orthopaedic Journal of Sports Medicine. (2018) 6:2325967118785365. doi: 10.1177/2325967118785365

19. Han F, Kong C, Yaser Hasan M, Ramruttun A, Kumar V. Superior Capsular Reconstruction for Irreparable Supraspinatus Tendon Tears Using the Long Head of Biceps: A Biomechanical Study on Cadavers. Orthop J Sports Med. (2019) 105:257-63. doi: 10.1016/j.otsr.2018.10.023

20. Lin J, Qi W, Liu Z, Chen Z, Li X, Yan Y, et al. An arthroscopic technique for full-thickness rotator cuff repair by transposition of the long head of biceps. Orthop Traumatol Surg Res. (2019) 105:265-9. doi: 10.1016/j.otsr.2018.07.027

21. Park M, Itami Y, Lin C, Kantor A, McGarry M, Park C, et al. Anterior cable reconstruction using the proximal biceps tendon for large rotator cuff defects limits superior migration and subacromial contact without inhibiting range of motion: a biomechanical analysis. Arthroscopy. (2018) 34:2590600. doi: 10.1016/j.arthro.2018.05.012
22. Mihata T, McGarry M, Kahn T, Goldberg I, Neo M, Thay Q. Biomechanical effect of thickness and tension of fascia lata graft on glenohumeral stability for superior capsule reconstruction in irreparable supraspinatus tears. Arthroscopy. (2016) 32:418-26. doi: 10.1016/j.arthro.2015. 08.024

23. Mihata T, Bui C, Masaki A, Cavagnaro M, Kuenzler M, Peterson A, et al. A biomechanical cadaveric study comparing superior capsule reconstruction using fascia lata allograft with human dermal allograft for irreparable rotator cuff tear. J Shoulder Elbow Surg. (2017) 26:215866. doi: 10.1016/j.jse.2017.07.019

24. Ciampi P, Scotti C, Nonis A, Vitali M, Di Serio C, Peretti G, et al. The benefit of synthetic versus biological patch augmentation in the repair of posterosuperior massive rotator cuff tears: a 3-year follow-up study. Am J Sports Med. (2014) 42:1169-75. doi: 10.1177/0363546514525592

25. Singh S, Reeves JG, Daniel G, Langohr JA, Athwal JG. The subacromial balloon spacer versus superior capsular reconstruction in the treatment of irreparable rotator cuff tears: a biomechanical assessment. Arthroscopy. (2019) 35:382-9. doi: 10.1016/j.arthro.2018.09.016

26. Gupta A, Hug K, Boggess B, Gavigan M, Toth AP. Massive or 2-tendon rotator cuff tears in active patients with minimal glenohumeral arthritis: clinical and radiographic outcomes of reconstruction using dermal tissue matrix xenograft. Am J Sports Med. (2013) 41:872-9. doi: 10.1177/03635465124 75204

27. Abd Elrahman AA, Sobhy MH, Abdelazim H, Omar Haroun HK. Superior capsular reconstruction: fascia lata versus acellular dermal allograft: a systematic review. Arthrosc Sports Med Rehabil. (2020) 28:e389-e397. doi: 10.1016/j.asmr.2020. 03.002

28. Liu J, Hughes R, Smutz WP, Niebur G, Nan-An K. Roles of deltoid and rotator cuff muscles in shoulder elevation. Clinical Biomechanics. (1997) 12:32-8. doi: 10.1016/S0268-0033(96)00047-2

29. Mihata T, McGarry M, Kahn T, Goldberg I, Neo M, Thay Q. Biomechanical role of capsular continuity in superior capsule reconstruction for irreparable tears of the supraspinatus tendon. Am J Sports Med. (2016) 44:142330. doi: $10.1177 / 0363546516631751$

30. Lin C, Su F, Chang C, Hong C, Jou M, Lin C, et al. Effect of shoulder abduction on the fixation of humeral greater tuberosity fractures: a biomechanical study for three types of fixation constructs. J Shoulder Elbow Surg. (2015) 24:547-54. doi: 10.1016/j.jse.2014.09.032

Conflict of Interest: The authors declare that the research was conducted in the absence of any commercial or financial relationships that could be construed as a potential conflict of interest.

Publisher's Note: All claims expressed in this article are solely those of the authors and do not necessarily represent those of their affiliated organizations, or those of the publisher, the editors and the reviewers. Any product that may be evaluated in this article, or claim that may be made by its manufacturer, is not guaranteed or endorsed by the publisher.

Copyright (c) 2021 Luciani, Farinelli, De Berardinis and Gigante. This is an openaccess article distributed under the terms of the Creative Commons Attribution License (CC BY). The use, distribution or reproduction in other forums is permitted, provided the original author(s) and the copyright owner(s) are credited and that the original publication in this journal is cited, in accordance with accepted academic practice. No use, distribution or reproduction is permitted which does not comply with these terms. 